\title{
Advances in the treatment of monoclonal gammopaties:The emerging role of targeted therapy in plasma cell dyscrasias
}

\author{
Aldo M Roccaro' \\ Irene M Ghobrial' \\ Simona Blotta' \\ Steven P Treon' \\ Michele Malagola ${ }^{2}$ \\ Kenneth C Anderson' \\ Paul G Richardson' \\ Domenico Russo ${ }^{2}$ \\ 'Department of Medical Oncology, \\ Dana-Farber Cancer Institute \\ and Harvard Medical School, Boston, \\ MA, USA; ${ }^{2}$ Unit of Blood Diseases \\ and Cell Therapies, University of \\ Brescia Medical School, Brescia, Italy
}

\begin{abstract}
The paradigm for the treatment of monoclonal gammopaties has dramatically changed: therapeutic options in multiple myeloma (MM) have evolved from the introduction of melphalan and prednisone in the 1960s, high-dose chemotherapy and stem cell transplantation in the late 1980s and 1990s, to the rapid introduction of small novel molecules within the last seven years. Based on the understanding of the complex interaction of the MM cells with the bone marrow microenvironment and the signaling pathways that are dysregulated in this process, a number of novel therapeutic agents are now available. Specifically, three novel agents with a specific-targeted anti-MM activity, have been FDA-approved for the treatment of this disease, namely Bortezomib, thalidomide, and lenalidomide which are now all playing a key role in the treatment of MM. The success of targeted therapy in MM has since led to the development and investigation of more than 30 new compounds in this disease and in other plasma cell dyscrasias such as Waldenström's macroglobulinemia and primary amyloidosis, both in the preclinical settings and as part of clinical trials.
\end{abstract}

Keywords: monoclonal gammopaties, targeted therapies

\section{Introduction}

Monoclonal gammopaties represent a clinically heterogeneous group of diseases generally considered plasma cell dyscrasias and characterized by abnormal production of monoclonal (M) immunoglobulin, also called M-protein or M-component, produced by a clone that developed from a common progenitors in the B lymphocyte lineage. The M-component may be detected by electrophoresis as a band of resctricted migration in the serum or urine. They include monoclonal gammophaty of undetermined significance (MGUS), multiple myeloma (MM), Waldenström's macroglobulinemia (WM), primary (AL) amyloidosis, heavy chain diseases, cryoglobulinemia type I and type II, and other lymphoproliferative disorders.

In this review, the role of new targeted therapies available for monoclonal gammopaties will be discussed, focusing on MM, WM, and amyloidosis.

After almost forty years, the paradigm for the treatment of monoclonal gammopaties has dramatically changed: for example, therapeutic options in MM have evolved from the introduction of melphalan and prednisone in the 1960s, high-dose chemotherapy and stem cell transplantation in the late 1980s and 1990s, to the rapid introduction of small novel molecules within the last seven years. Based on the understanding of the complex interaction of the MM cells with the bone marrow (BM) microenvironment and the signaling pathways that are dysregulated in this process, a number of novel therapeutic agents are now available. Specifically, three novel agents with a specific-targeted anti-MM activity, have been US Food and Drug Administration 
(FDA)-approved for the treatment of this disease, namely Bortezomib, thalidomide, and lenalidomide which are now all playing a key role in the treatment of MM. The success of targeted therapy in MM has since led to the development and investigation of more than 30 new compounds in this disease and in WM, both in the preclinical settings and as part of clinical trials.

\section{Immunomodulatory drugs (IMiDs): thalidomide and lenalidomide Thalidomide}

Thalidomide was first used as a sedative and hypnotic drug in the 1950's. It was withdrawn from the market because of its teratogenic effects. In 1999 a phase II study showed that thalidomide, used as a single agent in patients with relapsed MM, resulted in an overall response rate (ORR) of $25 \%$ (Singhal et al 1999). The main activity and efficacy of thalidomide in MM was then elucidated. It has been shown that thalidomide induces in vitro growth arrest, blocks the increased secretion of tumor necrosis factor alpha (TNF- $\alpha$ ), and affects the interaction between myeloma cells and BM microenvironment by decreasing the expression of adhesion molecules (E-selectin, L-selectin, ICAM-1, VCAM-1) or inhibiting the paracrine loops of cytokine secretion, such as vascular endothelial growth factor (VEGF) and interleukin (IL)-6; inhibits angiogenesis; and enhances host immune response against MM; interferes with intracellular growth signalling by inhibiting the constitutive activity of nuclear factor kappa B (NFkB) (Hideshima et al 2000; Davies et al 2001; Mitsiades et al 2002) (Figure 1). Several studies then tested the combination of thalidomide with other agents such as dexamethasone and chemotherapeutic drugs in patients with relapsed/refractory MM, and this led to response rates as high as 65\% (Rajkumar et al 2000, 2002; Weber et al 2003; Kumar et al 2006). After these encouraging results, thalidomide in combination with dexamethasone entered several phase II clinical trials in newly diagnosed MM patients, and demonstrated a RR of $\sim 65 \%$ (Rajkumar et al 2002; Weber et al 2003a; Kumar and Rajkumar 2006). Subsequently, a large phase III clinical trial was performed using thalidomide with dexamethasone versus high-dose dexamethasone alone for newly diagnosed MM patients, resulting in a $63 \% \mathrm{RR}$ in the thalidomide/dexamethasone arm versus $41 \%$ in the dexamethasone arm, although no survival advantage was observed between the two groups (Rajkumar et al 2006).

Other phase III trials in elderly patients who were not candidates for autologous stem cells transplant included a randomized study compared melphalan prednisone and thalidomide (MPT) versus melphalan and prednisone (MP), which showed that patients treated with MPT had higher RR (76\% versus 48\%) and longer event-free survival (EFS) than patients treated with MP alone (54\% versus 27\%) (Palumbo et al 2006). Facon and colleagues (2006) conducted a large phase III trial of MPT compared to MP or high dose chemotherapy and stem cell transplantation in elderly patients between 65 to 75 years of age and showed that patients treated with MPT had a longer overall survival of 54 months compared to 32 months for MP and 39 months for transplant.

A randomized study has recently investigated the activity of thalidomide in combination with VAD and doxil, compared to VAD-doxil and it resulted in a higher RR in the arm with thalidomide versus the arm without thalidomide $(81 \%$ versus 66\%) (Zervas et al 2006). The toxicities of thalidomide correlate both with dose and length of treatment and include neuropathy and deep vein thrombosis. Other important toxicities include fatigue, somnolence, constipation, rash (including Stevens-Johnson syndrome), and hepatic dysfunction (Ghobrial and Rajkumar 2003).

In view of its success in the treatment of patients with MM, thalidomide has been tested alone in WM patients, demonstrating partial response in $25 \%$ of patients treated with single-agent thalidomide. Adverse effects were common and prevented dose escalation of thalidomide in $75 \%$ of patients. In addition, thalidomide in combination with dexamethasone and clarithromycin induced partial response in 10 of $12(83 \%)$ previously treated patients (Dimopoulos et al 2003). However, a follow up study of 10 patients with higher doses of thalidomide (200 mg daily) showed only $20 \%$ overall response rate (Treon et al 2006a). Several clinical trials using thalidomide in combination with a wide variety of other compounds are ongoing in patients with $\mathrm{MM}$ and WM (Table 1).

High-dose chemotherapy has increased the response rate in patients with $\mathrm{MM}$, but this therapeutical option is not curative and an effective consolidation-maintenance could extend the duration of response. Several studies have evaluated the role of maintenance therapy with thalidomide in MM patients after autologous stem-cell transplantation, which shows that thalidomide improves survival and represents a valid and effective strategy as a maintenance therapy option (Attal et al 2006; Abdelkefi et al 2007; Spencer et al 2007).

Finally, thalidomide has been evaluated also in AL amyloidosis patients where it induced response rates up to 
Table I Ongoing clinical trials using thalidomide-based regimens in MM and WM (www.clinicaltrials.gov)

\begin{tabular}{|c|c|c|c|}
\hline Treatment regimen & Disease & Phase & Endpoints \\
\hline Thal+Bort & $\mathrm{MM} /$ refractory & I & Toxicities \\
\hline Thal+Dex vs Doxil+Thal+Dex & MM/newly diagnosed & III & ORR, OS \\
\hline Thal+Clarithromycin+Len+ Dex & $\mathrm{MM} /$ relapsed refractory & II & Safety, efficacy \\
\hline Thal+Doxorubicin+Dex & MM/untreated stage II/III & II & ORR, safety, toxicity \\
\hline Thal+/-Lenal+Dex & MM & III & ORR, toxicity \\
\hline Thal+Bort & MM/newly diagnosed stage II/III & II & $\begin{array}{l}\text { Tumor response, mobilization/ } \\
\text { collection stem cells, incidence/ } \\
\text { severity of neuropathy }\end{array}$ \\
\hline Thal+Doxil+Bort & MM/untreated stage I/II/III & II & ORR,TTP \\
\hline Thal $+\mathrm{AsO}_{3}+$ ascorbic acid+ Dex & MM/relapsed, refractory & I & Toxicity, safety, tolerability \\
\hline Thal+Oblimersen+Dex & MM/relapsed, refractory & II & ORR \\
\hline Thal+Bort+CTX+Dex & MM/untreated & II & ORR, safety, tolerability \\
\hline Thal+Riuximab & WM & ॥ & ORR, TTF, toxicity \\
\hline
\end{tabular}

Abbreviations: Thal, thalidomide; Bort, Bortezomib; Doxil, liposomal doxorubicine; Len, lenalidomide; Dex, dexamethasone; CTX, cyclophosphamide; As ${ }_{3}$, arsenic trioxide; Mel, melphalan; Pdn, prednisone; ORR, overall response rate; OS, overall response;TTP, time to progression; PFS, progression-free survival;TTF, time to treatment failure.

$50 \%$ when combined with dexamethasone. Unfortunately, the regimen is poorly tolerated, with $50 \%-65 \%$ of patients experiencing grade 3 or 4 toxicities (Palladini et al 2005).

\section{Lenalidomide}

Based on the success of thalidomide, lenalidomide (CC-5013; IMiD-3, Celgene Corp), a more potent immunomodulatory derivative of thalidomide was developed. Lenalidomide overcomes growth and survival advantage conferred by the BM-milieu, downregulates VEGF, and exerts antiangiogenic activities. In addition, lenalidomide co-stimulates $\mathrm{T}$ cells, enhances antitumor immunity mediated by interferon (IFN) $\gamma$ and IL-2, and augments natural killer (NK) cell cytotoxicity (Hideshima et al 2001; Mitsiades et al 2002; Dredge et al 2002) (Figure 1).

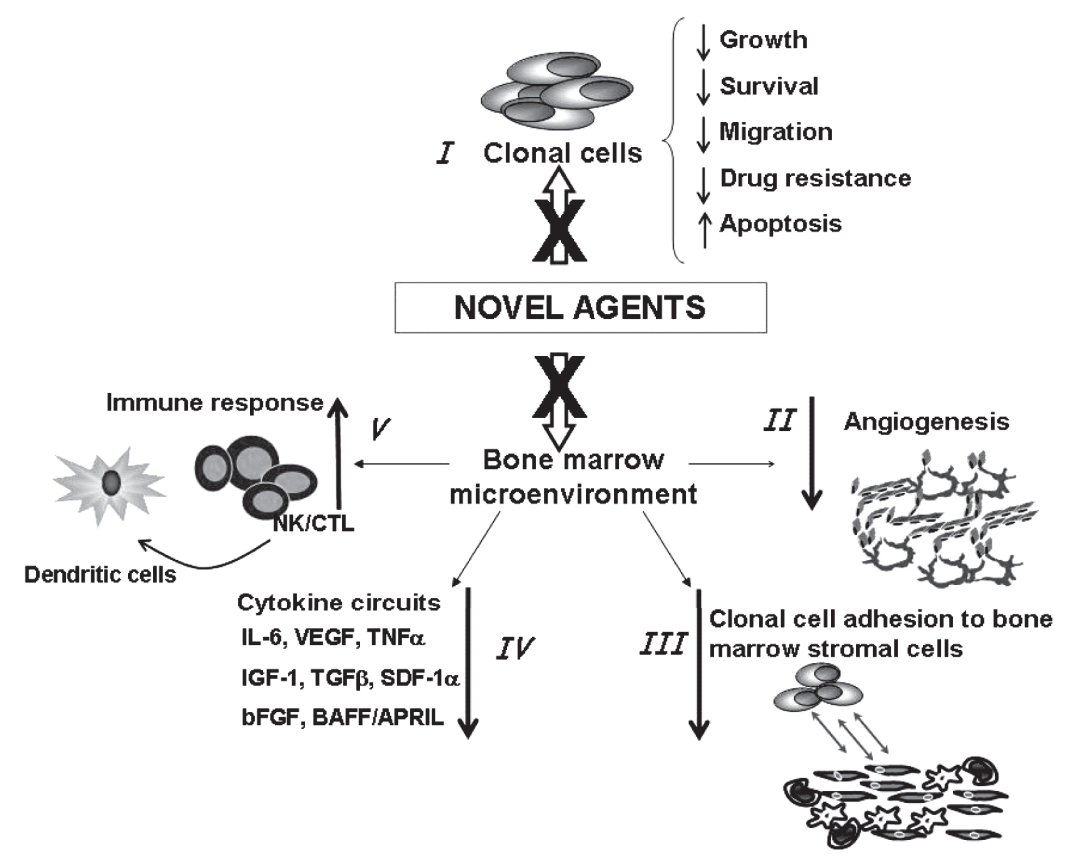

Figure I Mechanisms of action of novel agents. Novel molecules can: I) directly inhibit clonal cells; II) inhibit angiogensis; III) inhibit tumor cell adhesion to bone marrow stromal cells (BMSCs); IV) decrease cytokine production from BMSCs; V) increase host anti-tumor immunity. 
Phase I clinical trials using lenalidomide in patients with relapsed and refractory $\mathrm{MM}$, established a dose of $25 \mathrm{mg}$, and demonstrated a promising RR of 35\% (Richardson et al 2006a). Phase II studies followed and established the optimal schedule of 3 weeks on and 1 week off with once daily dosing (Richardson et al 2001, 2006b).

Then, two large randomized phase III studies (MM-009, MM-010) compared lenalidomide and dexamethasone to dexamethasone and placebo for patients with relapsed or relapsed and refractory MM. They both showed comparably favorable results, with RR and time to progression with the lenalidomide/dexamethasone combination significantly greater and more than twice the RR seen with dexamethasone alone (Dimopoulos 2005a; Weber et al 2006).

Based upon the success of these studies, lenalidomide received FDA-approval for the treatment of relapsed MM in June 2006. A phase II study of the combination of lenalidomide and dexamethasone was performed in 32 newly diagnosed patients with MM and showed an ORR of 91\% (Rajkumar et al 2005). A recent study demonstrated the efficacy of lenalidomide in combination with melphalan and prednisone which was associated with a RR of $86 \%$ (Palumbo et al 2006). Similarly, the combination of lenalidomide with other drugs such as adriamycin and dexamethasone showed a RR of 84\% (Knop et al 2006).

A Phase III clinical trial using lenalidomide in combination with dexamethasone in newly diagnosed MM patients has been recently completed and showed that lenalidomide plus low-dose dexamethasone is associated with superior OS compared to lenalidomide plus high-dose dexamethasone (Rajkumar et al 2007). The main side effects of lenalidomide include myelosuppression, particularly neutropenia and thrombocytopenia, and deep venous thrombosis especially when it used in combination with high-dose dexamethasone (Rajkumar and Blood 2006).

Based on the potent activity of lenalidomide in MM and considered the lack of neuropathy with this agent, a phase II study of lenalidomide $25 \mathrm{mg}$ daily in combination with rituximab is ongoing in patients with relapsed or relapsed/ refractory WM.

More than forty clinical trials using lenalidomide in combination with several other compound are actually on going in patients with MM and WM (Table 2).

Lenalidomide has also entered a phase II clinical trials for patients with AL amyloidosis. When combined with dexamethasone, lenalidomide induced response rates of nearly $67 \%$, with $29 \%$ of hematologic complete response (Sanhorawala et al 2007).

\section{Proteasome inhibitors: Bortezomib and second-generation (NPI-0052; PRI7I) \\ Bortezomib}

Bortezomib (PS-341, Millennium Pharmaceuticals, Inc) represents the first in class proteasome inhibitor to have progressed into widespread clinical use in MM patients, based on preclinical data showing its in vitro and in vivo anti-tumor activity in MM cells, by inhibiting proliferation, inducing apoptosis and by targeting the BM microenvironment through its antiangiogenic activity and by inhibiting the binding of MM cells to the BM stromal cells (Figure 1). Bortezomib as single agent has been evaluated in patients with advanced, heavily pretreated MM in the SUMMIT study (Study of Uncrontrolled Multiple Myeloma managed

Table 2 Ongoing clinical trials using lenalidomide-based regimens in MM and WM (www.clinicaltrials.gov)

\begin{tabular}{llll}
\hline Treatment regimen & Disease & Phase & Endpoints \\
\hline Len+Doxorubicine+Dex & MM/relapsed, refractory & I/II & Safety, efficacy, ORR \\
Len+Mel+Pdn vs high-dose Mel & MM/newly diagnosed & III & PFS, ORR, OS \\
Len+Bort+Dex vs & MM/untreated & I/II & ORR \\
Len+Bort+Dex+CTX & & & \\
Len+Dex+Thal & MM/Dex previously treated & III & PFS, ORR, OS \\
Len+Dex+Thal & MM/relapsed, refractory & II & Efficacy, safety \\
Len+Perifosine+Dex & MM & I & Safety, adverse events \\
Len+SGN-40+Dex & MM & I & safety \\
Len+Dex vs Dex & MM/previously treated & III , OS \\
Len+Mel+Pdn & MM/untreated patients & I/II & Toxicity,TTP, OS, ORR \\
Len+Mel & MM/untreated patients & II & Toxicity, tumor response, \\
Len+Rituximab & & II & TTP, OS, DFS \\
\hline
\end{tabular}

Abbreviations: Len, lenalidomide; Dex, dexamethasone; Mel, melphalan; Pdn, prednisone; Bort, Bortezomib; CTX, cyclophosphamide; Thal, thalidomide; ORR, overall response rate; PFS, progression-free survival; OS, overall survival;TTP: time to progression; DFS, disease-free survival. 
with proteasome Inhibition Therapy) (Richardson et al 2003) which showed an ORR of 35\% in 202 patients with relapsed and refractory MM. The CREST (Clinical Response and Efficacy Study of Bortezomib in the Treatment of myeloma) trial, a phase II study randomizing patients to higher $\left(1.3 \mathrm{mg} / \mathrm{m}^{3}\right)$ or lower $\left(1.0 \mathrm{mg} / \mathrm{m}^{3}\right)$ doses of Bortezomib in combination with dexamethasone, revealed positive response rates $(33 \%$ with low-dose Bortezomib alone, 44\% with low-dose Bortezomib/dexamethasone, 50\% with high-dose Bortezomib, and $62 \%$ with high-dose Bortezomib/dexamethasone) (Jagannath et al 2004). Subsequently, the APEX study (Assessment of Proteasome Inhibition for Extending Remission) compared Bortezomib with high-dose dexamethasone in patients with relapsed/refractory MM, and showed an ORR of 38\% in the Bortezomib arm, versus $18 \%$ obtained in the high-dose dexamethasone. Moreover, Bortezomib demonstrated superiority over dexamethasone in terms of time to progression and survival (Richardson et al 2005a). Based on these encouraging data, Bortezomib was FDA-approved in 2003 with full approval in 2005 and numerous trials using Bortezomib in combination with other agents were built.

Other combinations included chemotherapies and novel agents (Richardson et al 2006c). The combination of Bortezomib, thalidomide and dexamethasone (VTD) in patients with relapsed MM showed an overall response rate of $70 \%$ including near complete responses in 16\%. High responses were also observed in studies of patients with previously untreated MM. Single agent Bortezomib showed an overall response rate of $40 \%$ with $10 \%$ complete responses in a phase II study of 66 patients with MM.

The combination of Bortezomib and dexamethasone led to an overall response rate of $66 \%$ to $88 \%$ in another phase II trial of newly diagnosed MM (Jagannath et al 2006; Harousseau et al 2006).

In addition, the combination of Bortezomib (V), melphalan (M) and prednisone (P) (MPV) in nontransplant candidates resulted in an overall response rate of $89 \%$ (Mateos et al 2007). Interestingly, a phase III trial randomizing newly diagnosed MM patients to either VMP or MP, has been recently completed and showed that VMP significantly prolongs survival and is superior for all efficacy endpoints: specifically VMP induced rapid and durable responses with unprecedented complete response rate (35\%); prolonged time to progression ( $\sim 52 \%$ reduced risk of progression), time to next therapy/treatment free interval; and overall survival ( $\sim 40 \%$ reduced risk of death) (San Miguel et al 2007).

Also the combination of Bortezomib, dexamethasone, and cyclophosphamide was shown to be more effective than Bortezomib either used as single agent or with dexamethasone (Davies et al 2006). These encouraging results were subsequently confirmed by a multicenter randomized phase 3 study comparing the combination of doxil and Bortezomib versus Bortezomib alone (Orlowski et al 2006). Similarly it has been recently demonstrated that liposomal doxorubicin+Bortezomib significantly improves TTP compared to Bortezomib alone, regardless of the number of prior lines of therapy, or anthracycline exposure (Blade et al 2007).

Based on its activity in MM, single agent Bortezomib was tested in WM in phase II trials and achieved 40\%-80\% responses (Dimopoulos et al 2005b). The combination of Bortezomib, dexamethasone and rituximab was recently evaluated in untreated patients with WM. Each cycle of therapy consisted of IV Bortezomib at $1.3 \mathrm{mg} / \mathrm{m}^{2}$ and IV dexamethasone $40 \mathrm{mg}$ on days (1, 4, 8, and 11), and rituximab at $375 \mathrm{mg} / \mathrm{m}^{2}$ (day 11). Patients received four consecutive cycles, followed by a three-month pause, and then 4 more cycles, each given three months apart. The interim analysis of the first 10 patients who received the first 4 cycles of therapy showed partial response in 50\% and minor response in the other $50 \%$, with 2 patients $(20 \%)$ achieving an unconfirmed complete response (Treon et al 2006b).

There are actually several clinical trials ongoing using Bortezomib either alone or in combination with other agents in $\mathrm{MM}$ and WM patients (Table 3).

Recently the role of proteasome inhibition has been studied in AL amyloidosis, characterized by the overproduction of a destabilized light chain which tends to aggregate and deposit in several tissues (Sitia et al 2007; Kastritis et al 2007). The process of amyloid deposition induces tissue damage and subsequently organ failure, leading to high mortality. The combination of Bortezomib and dexamethasone has been successfully evaluated in patients with AL amyloidosis who were relapsed or progressed after previous thalidomide-based treatments, and who were ineligible for high-dose melphalan supported by autologous stem cell transplantation: 94\% hematologic responses were observed, including 44\% complete responses.

\section{New proteasome inhibitor, NPI-0052}

Based on the significant anti-MM activity of Bortezomib, a new proteasome inhibitor (NPI-0052; Nereus Pharmaceuticals, CA) with a different structure and different mechanism of action has been developed. NPI-0052 is an oral proteasome inhibitor that has shown significant anti-neoplastic activity in MM and WM (Chauhan et al 2005). Importantly, 
Table 3 Ongoing clinical trials using Bortezomib-based regimens in MM and WM (www.clinicaltrials.gov)

\begin{tabular}{|c|c|c|c|}
\hline Treatment regimen & Disease & Phase & Endpoints \\
\hline Bort + Perifosine $+/-$ Dex & $\begin{array}{l}\text { MM/relapsed, refractory, previously } \\
\text { treated with Bortezomib }\end{array}$ & $1 / I I$ & Toxicity, ORR \\
\hline Bort $+\mathrm{CCl}-779$ & $\mathrm{MM} /$ relapsed, relapsed/refractory & $\mathrm{I} / \mathrm{II}$ & Safety, MTD, TTP, PFS, OS \\
\hline $\begin{array}{l}\text { Bort+Doxil+Dex followed by } \\
\text { Thal+Dex +/- Bort }\end{array}$ & MM & II & Toxicity, disease response \\
\hline Bort+Sorafenib & MM/relapsed, refractory & $\mathrm{I} / \mathrm{II}$ & ORR, PFS \\
\hline Bort+Doxil+Mel & $\mathrm{MM} /$ relapsed, refractory & $\mathrm{I} / \mathrm{II}$ & Safety, tolerability, MTD \\
\hline Bort+Doxil & MM & II & ORR, toxicity, OS, PFS, TTP \\
\hline Bort $+\mathrm{AsO}_{3}+$ Vitamin C & MM & $1 / I I$ & Safety, toxicity, \\
\hline Bort+Samarium I 53 & MM/relapsed, refractory & 1 & Toxicity, MTD, TTP, PFS, OS \\
\hline Bort+Vorinostat (SAHA) & MM/relapsed, refractory & 1 & MTD, toxicity \\
\hline Bort+Ascorbic Acid+Mel & MM/newly diagnosed & II & $\begin{array}{l}\text { Safety, tolerability, ORR, TTP,TTR, } \\
\text { PFS, OS }\end{array}$ \\
\hline Bort+Dex+Doxil followed by CTX & $\begin{array}{l}\text { MM/post first line therapy; } \\
\text { relapsed/refractory Bortezomib } \\
\text { naïve }\end{array}$ & II & Efficacy, safety \\
\hline Bort+Thal+Dex & MM/relapsed, refractory & II & ORR, OS, PFS, toxicity \\
\hline $\begin{array}{l}\text { Bort+Dex+Thal vs } \\
\text { Bort+Dex+Thal+CTX }\end{array}$ & $\begin{array}{l}\text { MM/newly diagnosed candidate for } \\
\text { high-dose therapy and stem cell } \\
\text { transplantation }\end{array}$ & II & ORR,TTP, PFS \\
\hline Bort & WM & II & ORR, safety, tolerability \\
\hline Bort+Rituximab & WM/relapsed, refractory & II & ORR, safety, TTP \\
\hline Bort+Rituximab & WM/newly diagnosed & II & $\begin{array}{l}\text { ORR, ability to collect stem cells, } \\
\text { ORR,TTP, toxicity }\end{array}$ \\
\hline $\begin{array}{l}\text { Bort+Thal+Dex vs Bort+Dex vs } \\
\text { Bort+Mel+Pdn }\end{array}$ & MM/untreated & III & PFS \\
\hline Bort+Thal+Dex +/-Adriamycin & MM/relapsed, refractory & III & Efficacy, toxicity \\
\hline Bort+Dex+Rituximab & WM & II & Safety, tolerability, ORR,TTP \\
\hline Bort & WM/untreated or relapsed & II & $\begin{array}{l}\text { Toxicity, ORR, TTP, stable disease } \\
\text { duration, response duration }\end{array}$ \\
\hline
\end{tabular}

Abbreviations: Bort, Bortezomib; Dex, dexamethasone; Doxil, liposomal doxorubicine; Thal, thalidomide; CTX, cyclophosphamide; Mel, melphalan; AsO ${ }_{3}$, arsenic trioxide; ORR, overall response rate; MTD, maximum tolerated dose;TTP, time to progression; PFS, progression-free survival; OS, overall response.

the combination of NPI-0052 and Bortezomib induced significant inhibition of proliferation compared to each agent alone (Chauhan et al 2007; Roccaro et al 2008). A phase I clinical trial of NPI-0052 in relapsed MM has recently been initiated.

\section{PR-I7I}

PR-171 is a novel irreversible proteasome inhibitor under investigation for the treatment of hematological malignancies. Two phase I dose-escalation studies have been initiated, aimed at determining the safety, tolerability, and clinical response to PR-17 (O'Connor et al 2006). Patients with multiple myeloma, non-Hodgkin lymphoma, Hodgkin disease, or Waldenström macroglobulinemia who received two or more prior treatments were eligible. Two different dose-intensive schedules were employed in these phase I studies. PR-171 was well-tolerated, and several subjects have achieved longlasting $\mathrm{SD}$, reduction in paraprotein levels, or symptomatic improvement (O'Connor et al 2006).

\section{Signaling pathway inhibitors}

Preclinical data have been demonstrated that monoclonal gammopaties are characterized by disregulation of several signalling pathways, as compared to normal plasma cells (Hideshima et al 2004a; Hatjiharissi et al 2007; Leleu et al 2007). Moreover there is strong evidence that BM-milieu supports the growth of the clonal cell population. Therefore, this important knowledge has led to the development of several agents that specifically target the neoplastic clone by acting through those upregulated signaling pathways, the 
BM microenvironment, are able to affect both the clonal cells and the BM-milieu (Hideshima et al 2006).

\section{Signaling pathway inhibitors active in both MM and WM}

\section{Akt inhibitor: perifosine}

Perifosine (NCS-639966; Keryx Biopharmaceuticals, Inc) is an orally-active alkyl-phosphocholine molecule that affects membrane permeability; phospholipid metabolism; as well as mitogenic signaling transduction induced by the PI3/Akt pathway (Hideshima et al 2006). It has been recently demonstrated that perifosine has in vitro and in vivo activity against MM and WM cell lines and patient primary tumor cells, even in presence of BM stromal cells which are known to support tumor cell growth and induce resistance to apoptosis. In addition, perifosine showed synergistic activity when used in combination with other agents widely used in MM and WM such as dexamethasone, Bortezomib, doxorubicin, melphalan, and rituximab, specifically for MM and WM, respectively (Hideshima et al 2006; Leleu et al 2007). A phase II clinical trial of perifosine with or without dexamethasone in patients with relapsed and refractory MM has recently reported and showed activity, with $69 \%$ of patients achieving response and/or stabilization of disease (Richarson et al 2006d).

Another phase II trial of the combination of perifosine with Bortezomib \pm dexamethasone is currently underway in MM patients. Similarly, a phase II trial of single agent perifosine in patients with relapsed or relapsed/refractory WM has been initiated using $150 \mathrm{mg}$ oral daily dosing. The preliminary data of 13 patients enrolled on the study, with a median follow up time of 3 months, demonstrated promising activity of this agent. The treatment was well tolerated with minimal side effects. Seven patients were evaluable at the time of analysis and all showed evidence of $\operatorname{IgM}$ reduction, with a median IgM reduction of $14 \%(0 \%-25 \%)$. One patient whose IgM rose in the first month had a $50 \%$ reduction from the peak of IgM level at 3 months, indicating a delayed response. These preliminary results indicate that perifosine is a promising agent to be used in combination in future studies both in MM and WM.

\section{Protein kinase $\mathrm{C}$ inhibitor: enzastaurin}

Enzastaurin[H-Pyrrole-2,5-dione,3-(1-methyl-1H-indol3-yl)-4-[1-[-1(2pyridinylmethyl)-4-piperidinyl]-1H-indol3-yl], LY 317615; Eli Lilly and company, (Indianapolis, IN) is an oral PKC $\beta$ inhibitor, with downstream inhibition of Akt (Podar et al 2006). In MM, enzastaurin has demonstrated specific inhibition of $\mathrm{PKC}$ isoforms and Akt activation along with inducing cytotoxicity and apoptosis in MM and WM cells in vitro and in vivo (Podar et al 2006; Moreau et al 2007). Synergism has been demonstrated when enzastaurin was used in combination with Bortezomib. In addition, enzastaurin inhibted MM and WM cell growth in an in vivo xenograft model of these diseases. Based on these exciting preclinical data, enzastaurin alone and in combination with Bortezomib entered clinical trials in MM, and phase II trial are planned in WM as single agent.

\section{Mammalian target of rapamycin inhibitors: CCI-779, RAD00I}

mTOR inhibitors such as rapamycin and rapamycin analogues including CCI-779 and RAD001 have demonstrated in vitro and in vivo activity in MM cell lines and animal model (Shi et al 2002; Mitsiades et al 2004). The combination of rapamycin with active agents in MM such as lenalidomide, Bortezomib and 17-AAG have demonstrated synergistic activity in vitro (Raje et al 2004; Francis et al 2006). In addition, rapamycin appears to target the BM microenvironment by inhibiting angiogenesis and osteoclast formation in MM in vitro (Francis et al 2006). Similarly, Preclinical data have demonstrated increased activity of the PI3K/mTOR pathway in WM, and subsequently rapamycin (mTOR inhibitor) has been studied in vitro in WM and showed significant cytotoxicity in WM cells lines, specifically when combined with Bortezomib (unpublished data).

These findings have led to the design of studies using these agents in combination with other active agents in MM and WM. A phase II trial of RAD-001 in combination with lenalidomide, and a phase I/II clinical trial of CCI-779 in combination with Bortezomib are underway in patients with relapsed/refractory MM. In addition, a phase II trial of single agent RAD001 was initiated in aggressive, low grade lymphomas, and rare lymphomas including WM.

\section{Signaling pathway inhibitors active in MM MEK/ERK inhibitor:AZD-6244}

AZD6244 (AstraZeneca, Los Angeles, CA) has been tested in preclinical models in $\mathrm{MM}$ and induced inhibition of growth and cytotoxicity in MM cells even in the presence of cytokines/growth factors such as IL-6, IGF-1 that induce MEK/ERK activation (Hu et al 2003; Hideshima et al 2004a). A phase II trial of single agent AZD6244 is planned in 2007 for patients with relapsed/refractory MM. 


\section{P38MAPK inhibitor: SCIO-469}

SCIO-469 (Scios, Inc, Mountain View, CA) was first studied in clinical trials in rheumatoid arthritis and has shown in vitro activity in MM cells when co-cultured with BM stromal cells. The combination of SCIO-469 and Bortezomib demonstrated synergistic activity in vitro and in vivo (Hideshima et al 2004b). A phase II trial of SCIO-469 alone or in combination with Bortezomib in patients with relapsed MM showed stable disease in $24 \%$ with single agent SCIO- 469 , with its combination with Bortezomib resulting in a response rate of $32 \%$, including response in patients in whom Bortezomib had failed (Siegel et al 2006).

\section{Inhibitors of heat-shock protein 90: 17 - AAG, KOS-953, IPI-504}

Heat shock protein 90 (HSP90) inhibitors such as geldanamycin and 17-allylamino-17-demethoxygeldanamycin (17AAG) bind to the N-terminal ATP-binding pocket of Hsp90 and inhibit the stress induced anti-apoptotic response in $\mathrm{MM}$ cells and have demonstrated in vitro and in vivo cytotoxic activity alone and in combination with other agents active in MM, specifically Bortezomib (Mitsiades CS et al 2006). Phase I clinical trials of KOS 953-a 17AAG derivative - in MM have shown good tolerability with disease stabilization and minor response in patients with relapsed and refractory MM. Other HSP90 inhibitors include IPI-504, which is also being tested in a phase I clinical trial in MM and has excellent tolerability but no responses at doses tested to date (Richardson et al 2005b). Excitingly, KOS-953 combined with Bortezomib has demonstrated responses even in Bortezimibresistant patients in an ongoing phase I/II trial in patients with relapsed and refractory MM (Chanan-Khan et al 2005). Phase III trials of this combination are planned.

\section{Monoclonal antibodies mainly active INWM}

\section{Monoclonal anti-CD20 antibody: rituximab}

Rituximab has become one of the main treatment options of patients with WM. Standard rituximab (4 weekly infusions of $375 \mathrm{mg} / \mathrm{m}^{2}$ ) has demonstrated at least a minor response in $52 \%$ of patients (Gertz et al 2004). Four weekly rituximab treatments repeated at 3 months triggered response rates of 44\%-48\% (Dimopoulos et al 2003; Treon et al 2005a). Polymorphisms in the Fc $\gamma$ RIIIA (CD16) receptor gene may affect response to rituximab in WM. The response to rituximab is delayed in most patients with a median time to partial response of 4 months and a median time to best response of 17 months (Treon et al 2005b). In addition, the IgM level may initially increase in response to rituximab, a phenomenon termed IgM flare that occurs in about $54 \%$ of patients (Ghobrial et al 2004; Treon et al 2004a). These levels may persist for up to 4 months and do not indicate treatment failure, but may necessitate plasmapheresis to reduce hyperviscosity. Some patients receive maintenance therapy with rituximab. Although the impact of this regimen on the time to progression has not been determined specifically in WM, it has prolonged time to progression in patients in patients with other low-grade lymphomas who received rituximab maintenance compared to those who did not (van Oers et al 2006). Rituximab may also be useful in treating patients with IgM autoantibody-related neuropathies (Renaud et al 2006). The use of radioimmunotherapy such as iodine ${ }^{131} \mathrm{I}$ tositumomab radioimmunotherapy in WM has been limited since the high level of BM involvement precludes their use. However, case reports have shown that these therapies may be effective in patients with WM who have $<25 \% \mathrm{BM}$ involvement (Tsai e al 2004).

\section{Combinations of alkylating agents, nucleoside analogs, and rituximab}

The addition of alkylating agents to nucleoside analogs is active against WM. For example, the combination of oral cyclophosphamide with subcutaneous cladribine in 37 newly diagnosed patients achieved $84 \%$ PR or more, with a median duration of response of 36 months (Weber et al 2003b). The combination of fludarabine and intravenous cyclophosphamide in 11 previously treated patients resulted in 55\% overall response. In another study of 49 patients, the combination of fludarabine plus cyclophosphamide induced $78 \%$ overall response, with median time to treatment failure was 27 months (Tamburini et al 2005). Hematologic toxicity was commonly observed, and 3 patients died of treatment-related toxicities. A phase II clinical trial of 60 patients with WM treated with cyclophosphamide, rituximab, and dexamethasone (DRC) demonstrated an overall response rate of $70 \%$, with $7 \%$ complete remission (Dimopoulos et al 2006). Treatment was well tolerated and the main toxicity observed was grade 3-4 neutropenia in $20 \%$ of the patients. The combination of rituximab, cladribine, and cyclophosphamide was tested in 17 previously untreated patients with WM and achieved at least a partial response in $94 \%$ of the patients, with complete response in $18 \%$ (Weber et al 2003b). The combination of rituximab and fludarabine was evaluated 
in $43 \mathrm{WM}$ patients, with an overall response rate of $91 \%$ and CR of $7 \%$ (Treon et al 2004b). In another study, the combination of fludarabine, cyclophosphamide and rituximab (FCR) was tested in 21 patients with WM who had at least 1-2 prior regimens of therapy; overall response rate was $52 \%$, with $5 \%$ complete remissions (Treon et al 2006c). In MM, rituximab is being tested as a single agent or in combination with chemotherapeutic agents with some modest results of $\sim 64 \%$ minor responses and stable disease in one study (Moreau et al 2006).

\section{Other monoclonal antibodies specifically active in WM Anti-CD52 alemtuzumab-IH (Campath)} CD52 is highly expressed on WM cells in the BM, and alemtuzumab induces cytotoxicity of WM cells in vitro. A phase II study of alemtuzumab in 25 patients with relapsed WM or newly diagnosed untreated WM showed an overall response rate of $76 \%$, including $8(32 \%)$ partial responses and $11(44 \%)$ minor responses. Hematological toxicities were common among previously treated (but not untreated) patients and included G3/4 neutropenia (39\%); thrombocytopenia (18\%); anemia (7\%). G3/4 nonhematological toxicity for all patients included dermatitis (11\%); fatigue (7\%); and infection (7\%). CMV reactivation and infection was commonly seen among previously treated patients. Three patients died due to therapy-related complications (Hunter et al 2006).

\section{Anti-CD70 antibody: SGN-70}

Lymphoplasmacytic cells stimulate cell surface expression of TNF-family ligands through release of $\mathrm{sCD} 27$, which induces CD70 on mast cells. WM cells and cell lines highly express CD70 (Hatjiharissi et al 2006). Therefore, directly targeting CD70 using the fully humanized monoclonal antibody SGN-70 (Seattle Genetics, Inc., Bothell, WA) may represent a therapeutic option in WM. SGN-70 mediated significant dose-dependent ADCC against WM cell and mast cells at concentrations of $0.1-20 \mu \mathrm{g} / \mathrm{ml}$. SCID-hu mice bearing WM cells were treated with SGN-70 (1 mg/kg, i.p., qOD), and serum IgM and sCD27 levels were measured to monitor for disease progression. SGN-70 initiated 6 weeks following tumor engraftment blocked tumor growth in 12/12 treated mice, whereas all 5 untreated mice demonstrated disease progression (Hatjiharissi et al 2006). The results of these studies provide the framework for clinical trials to examine the therapeutic potential of the SGN-70 monoclonal antibody in WM.

\section{Other monoclonal antibodies} specifically active in MM

\section{SGN-40}

SGN-40 (Seattle Genetics, Inc.) is a humanized anti-CD40 ligand. It has been shown that CD40 induces proliferation of MM cells by activating the PI3/Akt pathway and by inducing secretion of IL-6 and VEGF from BM stromal cells (Tai et al 2005). Phase I study has been initiated demonstrating safety of this agent in MM with promising responses, and phase II trials are ongoing (Hussein et al 2006).

\section{mAb antiCD40 receptor: HCDI 22}

$\mathrm{HCD} 122$ is a a fully human, $\mathrm{IgG}_{1}$ antagonistic mAb targeting the CD40 receptor. A phase I trial has been conducted in MM patients and demonstrated that the Ab was safe, showing promising clinical activity in MM (Bensinger et al 2006).

\section{Oligonucleotide antisense, Bcl-2 antisense Bcl-2 inhibitor, G3I39 (Oblimersen sodium)}

Bcl-2 (Genasense, Genta Inc, Berkeley Heights, NJ) regulates apoptosis and resistance to chemotherapeutic agents; it has therefore become an attractive target for anticancer therapy in a number of malignancies including MM and WM (Chanan-Khan et al 2003). In vitro studies have shown that Bcl-2 is expressed in several B cell malignancies cells, and that downregulation of Bcl-2 and increased cytotoxicity in MM and WM cells may be achieved with G3139 (Badros et al 2005). A Phase I/II clinical trial of G3139 was conducted in patients with relapsed or relapsed/refractory WM showed favorable tolerability but little activity (Gertz et al 2005). Similarly, a phase II study of G3139 in combination with dexamethasone and thalidomide has been initiated in relapsed MM patients: the combination is well tolerated, and the responses are promising (Badros et al 2005).

\section{Other agents}

\section{Agents with preclinical activity in WM AMD3 100}

Waldenström's macroglobulinemia is characterized by widespread involvement of the BM, and lymphadenopathy in $20 \%$ of the patients, implying continuous trafficking of WM cells into and out of the BM and lymph nodes. The normal process of B-cell homing is regulated by cytokines, chemokines, and adhesion molecules (Lapidot et al 2005). One of the most extensively studied chemokines in migration is stromal 
derived factor SDF-1 and its receptor CXCR4. We recently demonstrated that WM cells and patient samples highly express CXCR4, and that SDF-1 induced migration of WM cells, with rapid activation of signaling pathways downstream of CXCR4 including pERK1/2, pAKT, and pPKC. The CXCR4 inhibitor AMD3100 (Genzyme, MA) inhibited migration of WM cells, as well as their adhesion to fibronectin. Adhesion of WM cells to stromal cells confers resistance to apoptosis and induces proliferation. The combination of AMD3100 with Bortezomib significantly enhances the cytotoxic effect of Bortezomib in the presence of stromal cells, possibly by interfering with adhesion of WM to stromal cells and thereby overcoming their protective effect (Ngo et al 2006). These studies provide the preclinical framework to study CXCR4 inhibitors in the regulation of homing and adhesion in WM.

\section{Triterpenoids, CDDO, and CDDO-Im}

2-cyano-3,12-dioxoolean-1,9-dien-28-oic acid (CDDO) and its methyl ester derivative (CDDO-Me) and imidazolide derivative (CDDO-Im) are synthetic triterpenoids derived from oleanolic acid. In vitro studies in primary WM samples showed that CDDO-Im inhibited cell proliferation and induced apoptosis in WM cells compared to normal B cells. There was evidence of PARP cleavage in a dose-dependent manner, suggesting that CDDO-Im induced malignant cell death occurs through a caspase-dependent mechanism, and may have potential efficacy in WM patients (Elsawa et al 2006).

\section{Simvastatin and resveratrol}

The antineoplastic activity of simvastatin and resveratrol in WM has been reported (Moreau et al 2007; Roccaro et al 2008). The two compounds exert antiproliferative activity and induce apoptosis in WM. Interestingly, they target WM cells even in the presence of bone marrow microenvironment and cytokines that are known to promote WM cell growth. Moreover, they both showed synergism when used in combination with other agents widely used in WM, such as dexamethasone and Bortezomib. Those preclinical in vitro data provide the framework for clinical trials of simvastatin or resveratrol in WM.

\section{Sildenafil citrate}

Based on the clinical observation that patients receiving sildenafil citrate had a decrease in their IgM (Treon et al 2004c), a phase II trial of single agent sildenafil citrate in patients with slowly progressing WM, who did not meet consensus eligibility for active therapy, was initiated. The purpose of the study was to delay time to progression in these patients. Thirty patients were treated on this study, and disease progression was suppressed in more than $50 \%$ of the patients. After 3 months of therapy, 63\% showed a decrease in IgM levels and $17 \%$ showed a minor response. However, disease progression at 6 months of follow occurred in almost all the patients (Patterson et al 2006).

\section{Imatinib mesylate}

Imatinib mesylate (Gleevec) targets the microenvironment of WM through inhibition of stem cell factor signaling through CD117, which is expressed on WM and mast cells. A phase II trial of single agent imatinib is ongoing in patients with relapsed or refractory WM. Imatinib is given at $400 \mathrm{mg}$ daily, with dose escalation to $600 \mathrm{mg}$ after one month of therapy. After 3 months of therapy, 6/13 (46.2\%) of patients achieved MR. The main toxicities observed included cytopenias, edema, and hyperglycemia, leading to dose reductions in $31 \%$ patients and cessation of therapy in 23\% patients (Treon et al 2006d).

\section{TACl-lg, Atacicept}

Atacicept (TACI-Ig; ZymoGenetics, Seattle, WI) contains the soluble TACI receptor that binds to the cytokines BLyS and APRIL, members of the tumor necrosis factor family that promote B-cell survival. An open-label, dose-escalation Phase $1 \mathrm{~b}$ study enrolled 16 patients with refractory or relapsed MM or active progressive WM. Sequential cohorts received one cycle of 5 weekly subcutaneous injections of atacicept at 2, 4, 7, or $10 \mathrm{mg} / \mathrm{kg}$. Treatment with atacicept was well tolerated, and no dose limiting toxicity was observed. A biological response was observed in this heavily treated refractory population, with disease stabilization in $75 \%$ of the patients with WM (Rossi et al 2006).

\section{Conclusions}

In summary, the last decade has marked a new era in the treatment of diseases characterized by monoclonal gammopaties. Indeed, a new paradigm shift has evolved utilizing novel therapeutic agents targeting the malignant clone and its bone marrow microenvironment. The combination of novel agents with chemotherapeutic drugs and/or glucocorticoids has demonstrated high response rates with complete remission rates comparable to those achieved in the stem cell transplant setting. This has been supported by in vitro and in vivo evidence showing the antitumor activity of those novel agents in MM, WM, as well as in other B cell maligancies. The future holds many more challenges for the treatment of MM and WM. These include combination of agents that achieve higher responses and longer survival, individualized therapies that are based on genetic and molecular abnormalities present in each patient, and clinical trials to test the benefit of novel 
agents in comparison and in addition to autologous stem cell transplantation, as well as other conventional approaches. Together, these therapies should lead to higher response rates, more durable duration of response, less toxicity and prolonged survival for patients, making plasma cell dyscrasias an increasingly chronic and treatable diseases.

\section{Disclosure}

Supported in part by Berlucchi Foundation (AMR) and Fondi 60\% 2006-2007.

\section{References}

Abdelkefi A, Ladeb S, Torjman L, et al. 2008. Single autologous stem cell transplantation followed by maintenance therapy with thalidomide is superior to double autologous transplantation in multiple myeloma: results of a multicentre randomized clinical trial. Blood, 111:1805-10.

Anderson K, Richardson P, Chanan-Khan A, et al. 2006. Single-agent Bortezomib in previously untreated multiple myeloma (MM): Results of a phase II multicenter study [abstract]. J Clin Oncol, 18S:7504.

Attal M, Harousseasu JL, Leyvraz S, et al. 2006. Maintenance therapy with thalidomide improves survival in multiple myeloma patients. Blood, 108:3289-94.

Badros AZ, Goloubeva O, Rapoport AP, et al. 2005. Phase II study of G3139, a Bcl-2 antisense oligonucleotide, in combination with dexamethasone and thalidomide in relapsed multiple myeloma patients. J Clin Oncol, 23:4089-99.

Bensinger W, Jagannath A, Becker P, et al. 2006. Phase I dose escalation study of a fully human, antagonist anti-CD40 antibody, HCD122 (Formerly CHIR-12.12) in patients with relapsed and refractory multiple myeloma [abstract]. Blood, 108:3575.

Blade J, San Miguel J, Nagler A, et al. 2007. The prolonged time to progression with pegylated liposomal doxorubicin + Bortezomib versus Bortezomib alone in relapsed or refractory multiple myeloma is unaffected by extent of prior therapy or previous anthracycline exposure [abstract]. Blood, 110:410.

Chanan-Khan AA. 2004. Bcl-2 antisense therapy in multiple myeloma. Oncology (Huntington), 18:21-4.

Chanan-Khan AA, Richardson PG, Alsina M, et al. 2005. Phase 1 clinical trial of KOS-953 + Bortezomib (BZ) in relapsed refractory multiple myeloma (MM) [abstract]. Blood, 106:362.

Chauhan D, Catley L, Li G, et al. 2005. A novel orally active proteasome inhibitor induces apoptosis in multiple myeloma cells with mechanisms distinct from Bortezomib. Cancer Cell, 8:407-19.

Chauhan D, Singh A, Brahmandam M, et al. 2007. Combination of proteasome inhibitors Bortezomib and NPI-0052 trigger in vivo synergistic cytotoxicity in multiple myeloma. Blood, 111:1654-64.

Davies FE, Raje N, Hideshima T, et al. 2001. Thalidimide and immunomodulatory derivatives augment natural killer cell cytotoxicity in multiple myeloma. Blood, 98:210-16.

Davies FE, Wu P, Srikanth M, et al. 2006. The combination of cyclophosphamide, velcade and dexamethasone (CVD) induces high response rates with minimal toxicity compared to velcade alone $(\mathrm{V})$ and velcade plus dexamethasone [abstract]. Blood, 108:3537.

Deocampo R, Rich R, Ryoo JJ, et al. 2002. Immunomodulatory drug CC5013 overcomes drug resistance and is well tolerated in patients with relapsed multiple myeloma. Blood, 100:3063-7.

Dimopoulos MA, Tsatalas C, Zomas A, et al. 2003. Treatment of Waldenström's macroglobulinemia with single-agent thalidomide or with the combination of clarithromycin, thalidomide and dexamethasone. Semin Oncol, 30:265-9.

Dimopoulos MA, Spencer A, Attal M, et al. 2005. Study of lenalidomide plus dexamethasone versus dexamethasone alone in relapsed or refractory multiple myeloma (MM): results of a phase 3 study (MM010) [abstract]. Blood, 106:6.
Dimopoulos MA, Anagnostopoulos A, Kyrtsonis MC, et al. 2005. Treatment of relapsed or refractory Waldenström's macroglobulinemia with Bortezomib. Haematologica, 90:1655-8.

Dimopoulos M, Anagnostopoulos A, Kyrtsonis M, et al. 2006. Primary treatment of Waldenströms macroglobulinemia (WM) with dexamethasone, rituximab and cyclophosphamide [abstract]. Blood, 108:128.

Dredge K, Marriott JB, Macdonald CD, et al. 2002. Novel thalidomide analogues display anti-angiogenic activity independently of immunomodulatory effects. Br J Cancer, 87:1166-72.

Elsawa S, Novak A, Konopleva M, et al. 2006. Preferential inhibition of malignant cell growth by CDDO in Waldenströms macroglobulinemia [abstract]. Blood, 108:2528.

Facon T, Mary J, Harousseau J, et al. 2006. Superiority of melphalanprednisone (MP) + thalidomide (THAL) over MP and autologous stem cell transplantation in the treatment of newly diagnosed elderly patients with multiple myeloma [abstract]. J Clin Oncol, 18S:1.

Francis LK, Alsayed Y, Leleu X, et al. 2006. Combination mammalian target of rapamycin inhibitor rapamycin and HSP90 inhibitor 17-allylamino17-demethoxygeldanamycin has synergistic activity in multiple myeloma. Clin Cancer Res, 12:6826-35.

Gertz MA, Geyer SM, Badros A, et al. 2005. Early results of a phase I trial of oblimersen sodium for relapsed or refractory Waldenström's macroglobulinemia. Clin Lymphoma, 5:282-4.

Gertz MA, Blood E, Kaminer LS, et al. 2004. Multicenter phase 2 trial of rituximab for Waldenström macroglobulinemia (WM): an Eastern Cooperative Oncology Group Study (E3A98). Leuk Lymphoma, 45:2047-55.

Ghobrial IM, Rajkumar SV. 2003. Management of thalidomide toxicity. $J$ Support Oncol, 1:194-205.

Ghobrial I, Fonseca R, Greipp PR, et al. 2004. Initial immunoglobulin M 'flare' after rituximab therapy in patients diagnosed with Waldenström macroglobulinemia: an Eastern Cooperative Oncology Group Study. Cancer, 101:2593-8.

Harousseau JL, Attal M, Leleu X, et al. 2006. Bortezomib plus dexamethasone as induction treatment prior to autologous stem cell transplantation in patients with newly diagnosed multiple myeloma: results of an IFM phase II study. Haematologica, 91:1498-505.

Hatjiharissi E, Ho A, Xu L, et al. 2006. Preclinical in vitro and in vivo evidence support a therapeutic role for the CD70 directed monoclonal antibody (SGN-70) in Waldenströms macroglobulinemia (WM) [abstract]. Blood, 108:2490.

Hatjiharissi E, Ngo H, Leontovic AA, et al. 2007. Proteomic analysis of Waldenström macroglobulinemia. Cancer Res, 67:3777-84.

Hideshima T, Chauhan D, Shima Y, et al. 2000. Thalidomide and its analogs overcome drug resistance of human multiple myeloma cells to conventional therapy. Blood, 96:2943-50.

Hideshima T, Chauhan D, Podar K, et al. 2001. Novel therapies targeting the myeloma cell and its bone marrow microenvironment. Semin Oncol, 28:607-12.

Hideshima T, Bergsagel PL, Kuehl WM, et al. 2004a. Advances in biology of multiple myeloma: clinical applications. Blood, 104:607-18.

Hideshima T, Podar K, Chauhan D, et al. 2004b. p38 MAPK inhibition enhances PS-341 (Bortezomib)-induced cytotoxicity against multiple myeloma cells. Oncogene, 23:8766-76.

Hideshima T, Catley L, Yasui H, et al. 2006. Perifosine, an oral bioactive novel alkylphospholipid, inhibts Akt and induces in vitro and in vivo cytotoxicity in human multiple myeloma cells. Blood, 107:4053-62.

Hu L, Shi Y, Hsu JH, et al. 2003. Downstream effectors of oncogenic ras in multiple myeloma cells. Blood, 101:3126-35.

Hunter Z, Boxer M, Kahl B, et al. 2006. Phase II study of alemtuzumab in lymphoplasmacytic lymphoma: results of WMCTG trial 02-079 [abstract]. J Clin Oncol, 24:7523.

Hussein M, Berenson J, Niesvisky R, et al. 2006. Results of a phase I trial of SGN-40 (Anti-huCd40 mAb) in patients with relapsed multiple myeloma [abstract]. Blood, 108:3576.

Jagannath S, Barlogie B, Berenson J, et al. 2004. A phase 2 study of two doses of Bortezomib in relapsed or refractory myeloma. Br J Haematol, $127: 165-72$ 
Jagannath S, Richardson PG, Barlogie B, et al. 2006. Bortezomib in combination with dexamethasone for the treatment of patients with relapsed and/or refractory multiple myeloma with less than optimal response to Bortezomib alone. Haematologica, 91:929-34.

Kastritis E, Anagnostopoulos A, Roussou M, et al. 2007. Treatment of light chain (AL) amyloidosis with the combination of Bortezomib and dexamethasone. Haematologica, 92:1351-58.

Knop S, Gerecke C, Topp M, et al. 2006. Lenalidomide (revlimid), adriamycin and dexamethasone chemotherapy (RAD) is safe and effective in treatment of relapsed multiple myeloma first results of a german multicenter Phase I/II trial [abstract]. Blood, 108:408.

Kumar S, Rajkumar SV. 2006. Thalidomide and lenalidomide in the treatment of multiple myeloma. Eur J Cancer, 42:1612-22.

Lapidot T, Dar A, Kollet O. 2005. How do stem cells find their way home? Blood 106:1901-10.

Leleu X, Xiaoying J, Runnels J, et al. 2007. The Akt pathway regulates survival and homing in Waldenström macroglobulinemia. Blood, 110:4417-26.

Mateos MV, Hernandez JM, Hernandez MT, et al. 2006. Bortezomib plus melphalan and prednisone in elderly untreated patients with multiple myeloma: results of a multicenter phase 1/2 study. Blood, 108:2165-72.

Mitsiades CS, Mitsiades N, Poulaki V, et al. 2002. Activation of NF-kap$\mathrm{paB}$ and upregulation of intracellular anti-apoptotic proteins via the IGF-1/Akt signalling in human multiple myeloma cells: therapeutic implications. Oncogene, 21:5673-83.

Mitsiades CS, Mitsiades NS, McMullan CJ, et al. 2006. Antimyeloma activity of heat shock protein-90 inhibition. Blood, 107:1092-100.

Mitsiades N, Mitsiades CS, Poulaki V, et al. 2002. Apoptotic signaling induced by immunomodulatory thalidomide analogs in human multiple myeloma cells: therapeutic implications. Blood, 99:4525-30.

Mitsiades N, McMullan C, Poulaki V, et al. 2004. The mTOR inhibitor RAD001 (Everolimus) is active against multiple myeloma cells in vitro and in vivo [abstract]. Blood, 104:1496.

Moreau AS, Jia X, Ngo HT, et al. 2007. Protein kinase C inhibitor enzastaurin induces in vitro and in vivo antitumor activity in Waldenström's macroglobulinemia. Blood, 109:4964-72.

Moreau AS, Jia X, Leleu X, et al. 2007. Simvastatin, an HMG-CoA inhibitor, induces in vitro antitumor activity in Waldenström's macroglobulinemia [abstract]. Hematologica, 92:1219.

Moreau P, Voillat 1, Benboubker L, et al. 2006. Rituximab in CD20 positive multiple myeloma: a prospective study from the IFM group [abstract]. Blood, 108:3577.

Ngo H, Hatjiharissi, E, Leleu, X, et al. 2006. The CXCR4/SDF-1 axis regulates migration and adhesion in Waldenström macroglobulinemia [abstract]. Blood, 108:2418.

O'Connor O, Orlowski, R, Alsina M, et al. 2006. Multicenter phase I studies to evaluate the safety, tolerability, and clinical response to intensive dosing with the proteasome inhibitor PR-171 in patients with relapsed or refractory hematological malignancies [abstract]. Blood, 108:2430.

Orlowski RZ, Zhuang SH, Parekh T, et al. 2006. The combination of pegylated liposomal doxorubicin and Bortezomib significantly improves time to progression of patients with relapsed/refractory multiple myeloma compared with Bortezomib alone: results from a planned interim analysis of a randomized phase III study [abstract]. Blood, 108:404.

Palladini G, Perfetti V, Obici L, et al. 2005. The combination of thalidomide and intermediate-dose dexamethasone is an effective but toxic treatment for patients with primary amyloidosis (AL). Blood, 105:2949-51.

Palumbo A, Bringhen S, Caravita T, et al. 2006. Oral melphalan and prednisone chemotherapy plus thalidomide compared with melphalan and prednisone alone in elderly patients with multiple myeloma: randomised controlled trial. Lancet, 367:825-31.

Palumbo A, Falco P, Falcone A, et al. 2006. Oral revlimid plus melphalan and prednisone (R-MP) for newly diagnosed multiple myeloma: Results of a multicenter phase I/II study [abstract]. Blood, 108:800.

Patterson C, Soumerai J, Hunter Z, et al. 2006. Sildenafil citrate suppresses disease progression in patients with Waldenström's macroglobulinemia. J Clin Oncol, 18S:7556.
Podar K, Raab MS, Zhang J, et al. 2006. Targeting PKC in multiple myeloma: in vitro and in vivo effects of the novel, orally available small-molecule inhibitor Enzastaurin (LY317615.HCl). Blood, 109:1669-77.

Raje N, Kumar S, Hideshima T, et al. 2004. Combination of the mTOR inhibitor rapamycin and CC-5013 has synergistic activity in multiple myeloma. Blood, 104:4188-93.

Rajkumar SV, Fonseca R, Dispenzieri A, et al. 2000. Thalidomide in the treatment of relapsed multiple myeloma. Mayo Clin Proc, 75:897-901.

Rajkumar SV, Hayman S, Gertz MA, et al. 2002. Combination therapy with thalidomide plus dexamethasone for newly diagnosed myeloma. J Clin Oncol, 20:4319-23.

Rajkumar SV, Hayman SR, Lacy MQ, et al. 2005. Combination therapy with lenalidomide plus dexamethasone (Rev/Dex) for newly diagnosed myeloma. Blood, 106:4050-3.

Rajkumar SV, Blood E, Vesole D, et al. 2006. Eastern Cooperative Oncology Group Phase III clinical trial of thalidomide plus dexamethasone compared with dexamethasone alone in newly diagnosed multiple myeloma: a clinical trial coordinated by the Eastern Cooperative Oncology Group. J Clin Oncol, 24:431-6.

Rajkumar SV, Blood E. 2006. Lenalidomide and venous thrombosis in multiple myeloma. N Engl J Med, 354:2079-80.

Rajkumar V, Jacobus S, Callander N, et al. 2007. A randomized trial of lenalidomide plus high-dose dexamethasone (RD) versus lenalidomide plus low-dose dexamethasone (Rd) in newly diagnosed multiple myeloma (E4A03): A trial coordinated by the Eastern Cooperative Oncology Group [abstract]. Blood, 110:74.

Renaud S, Fuhr P, Schweikert GM, et al. 2006. High-dose rituximab and anti-MAG-associated polyneuropathy. Neurology, 66:742-4.

Richardson PG, Barlogie B, Berenson J, et al. 2003. A phase 2 study of Bortezomib in relapsed, refractory myeloma. $N$ Engl $J$ Med, 348:2609-17.

Richardson PG, Schlossman RL, Weller E, et al. 2002. Immunomodulatory drug CC-5013 overcomes drug resistance and is well tolerated in patients with relapsed multiple myeloma. Blood, 100:3063-7.

Richardson PG, Sonneveld P, Schuster MW, et al. 2005a. Bortezomib or high-dose dexamethasone for relapsed multiple myeloma. $N$ Engl J Med, 352:2487-98.

Richardson P, Chanan-Khan A, Alsina M, et al. 2005b. Safety and activity of KOS-953 in patients with relapsed refractory multiple myeloma (MM): Interim results of a phase 1 trial [abstract]. Blood, 106:361.

Richardson PG, Mitsiades C, Hideshima T, et al. 2006a. Lenalidomide in multiple myeloma. Expert Rev Anticancer Ther, 6:1165-73.

Richardson PG, Blood E, Mitsiades CS, et al. 2006b. A randomized phase 2 study of lenalidomide therapy for patients with relapsed or relapsed and refractory multiple myeloma. Blood, 108:3458-64.

Richardson PG, Mitsiades C, Ghobrial I, et al. 2006c. Beyond single-agent Bortezomib: combination regimens in relapsed multiple myeloma. Curr Opin Oncol, 18:598-608.

Richardson P, Lonial S, Jakubowiak J, et al. 2006d. A multicenter phase II study of perifosine (KRX-0401) alone and in combination with dexamethasone (Dex) for patients with relapsed or relapsed/refractory multiple myeloma (MM) [abstract]. Blood, 108:3582.

Roccaro A, Leleu X, SAcco A, et al. 2008. Dual targeting of the proteasome regulates survival and homing in Waldenström's macroglobulinemia. Blood, 111:4752-63.

Roccaro A, Leleu X, Moreau AS, et al. 2008. Resveratrol exerts antiproliferative effect and induces apoptosis in Waldesntrom's macroglobulinemia. Clin Cancer Res, 14:1849-58.

Rossi J, Moreaux J, Rose M, et al. 2006. A Phase I/II study of atacicept (TACI-Ig) to neutralize APRIL and BLyS in patients with refractory or relapsed multiple myeloma (MM) or active previously treated Waldenström's macroglobulinemia (WM) [abstract]. Blood, 108:3578.

Sanchorawala V, Wright DG, Rosenzweig M, et al. 2007. Lenalidomide and dexamethasone in the treatment of AL amyloidosis: results of a phase 2 trail. Blood, 109:492-6. 
San Miguel J, Schlag R, Khuageva O, et al. 2007. MMY-3002: A phase 3 study comparing Bortezomib-melphalan-prednisone (VMP) with melphalan-prednisone (MP) in newly diagnosed multiple myeloma [abstract]. Blood, 110:76.

Shi Y, Gera J, Hu L, et al. 2002. Enhanced sensitivity of multiple myeloma cells containing PTEN mutations to CCI-779. Cancer Res, 62:5027-34

Siegel D, Krishnan A, Lonial S, et al. 2006. Phase II trial of SCIO-469 as monotherapy (M) or in combination with Bortezomib (MB) in relapsed refractory multiple myeloma (MM) [abstract]. Blood, 108:3580.

Singhal S, Mehta J, Desikan R, et al. 1999. Antitumor activity of thalidomide in refractory multiple myeloma. $N$ Engl J Med, 341:1565-71.

Sitia R, Palladini G, Merlini G. 2007. Bortezomib in the treatment of AL amyloidosis: targeted therapy? Haematologica, 92:1302-7.

Spencer A, Prince HM, Roberts A, et al. 2007. Thalidomide improve survivals when use after ASCT [abstract]. Hematologica, 92(suppl 2):S7b.5.

Tai YT, Tong X, Santos D, et al. 2005. Human anti-CD40 antagonist antibody triggers significant antitumor activity against human multiple myeloma. Cancer Res, 65:5898-906.

Tamburini J, Levy V, Chaleteix C, et al. 2005. Treatment of Waldenström's macroglobulinemia with the combination of fludarabine and cyclophosphamide: results in 49 patients. Leukemia, 19:1831-4.

Treon S, Branagan AR, Hunter Z, et al. 2004a. Paradoxical increases in serum IgM and viscosity levels following rituximab in Waldenström's macroglobulinemia. Ann Oncol, 15:1481-3.

Treon S, Branagan A, Wasi P, et al. 2004b. Combination therapy with rituximab and fludarabine in Waldenström's macroglobulinemia [abstract]. Blood, 104:753.

Treon SP, Tournilhac O, Branagan AR, et al. 2004c. Clinical responses to sildenafil in Waldenström's macroglobulinemia. Clin Lymphoma, 5:205-7.

Treon SP, Emmanouilides C, Kimby E, et al. 2005a. Extended rituximab therapy in Waldenström's macroglobulinemia. Ann Oncol, 16:132-8.

Treon S, Hansen M, Branagan AR, et al. 2005b. Polymorphisms in FcyRIIIA (CD16) receptor expression are associated with clinical response to rituximab in Waldenström's macroglobulinemia. $J$ Clin Oncol, 23:474-81.

Treon SP, Gertz MA, Dimopoulos M, et al. 2006a. Update on treatment recommendations from the Third International Workshop on Waldenström's macroglobulinemia. Blood, 107:3442-6.
Treon SP, Soumerai J, Patterson C, et al. 2006b. Bortezomib, dexamethasone and rituximab (BDR) is a highly active regimen in the primary therapy of Waldenström's macroglobulinemia: planned interim results of WMCTG clinical trial 05-180 [abstract]. Blood, 108:2765.

Treon SP, Gertz MA, Dimopoulos M, et al. 2006c. Update on treatment recommendations from the Third International Workshop on Waldenström's macroglobulinemia. Blood, 107:3442-6.

Treon SP, Soumerai J, Patterson C, et al. 2006d. Imatinib mesylate (Gleevec) is active in relapsed/refractory Waldenströms macroglobulinemia: Planned interim results of WMCTG Clinical Trial 05-140 [abstract]. Blood, 108:2484.

Tsai D, Maillard I, Downs LH, et al. 2004. Use of iodine 131I-tositumomab radioimmunotherapy in a patient with Waldenström's macroglobulinemia. Leuk Lymphoma, 45:591-5.

van Oers MH, Klasa R, Marcus RE, et al. 2006. Rituximab maintenance improves clinical outcome of relapsed/resistant follicular non-Hodgkin lymphoma in patients both with and without rituximab during induction: results of a prospective randomized phase 3 intergroup trial. Blood, 108:3295-301.

Weber D, Rankin K, Gavino M, et al. 2003a. Thalidomide alone or with dexamethasone for previously untreated multiple myeloma. J Clin Oncol, 21:16-19.

Weber DM, Dimopoulos MA, Delasalle K, et al. 2003b. 2-Chlorodeoxyadenosine alone and in combination for previously untreated Waldenström's macroglobulinemia. Semin Oncol, 30:243-7.

Weber D, Chen C, Niesvizky M, et al. 2006. Lenalidomide plus highdose dexamethasone provides improved overall survival compared to high-dose dexamethasone alone for relapsed or refractory multiple myeloma (MM): Results of a North American phase III study (MM009) [abstract]. J Clin Oncol, 18S:7521.

Zervas K, Mihou D, Katodritou I, et al. 2006. VAD-doxil vs VAD-doxil plus thalidomide as initial treatment in patients with multiple myeloma: a multicenter randomized trial of The Greek Myeloma Study Group [abstract]. Blood, 108:794. 
